\title{
BMJ Open Psychological factors and DNA methylation of genes related to immune/inflammatory system markers: the VA Normative Aging Study
}

Daniel Kim, ${ }^{1,2,3}$ Laura D Kubzansky, ${ }^{2}$ Andrea Baccarelli, ${ }^{4}$ David Sparrow, ${ }^{5,6}$ Avron Spiro III, ${ }^{6,7,8}$ Letizia Tarantini, ${ }^{9,10}$ Laura Cantone, ${ }^{9}$ Pantel Vokonas, ${ }^{6,7}$ Joel Schwartz ${ }^{4,11}$

To cite: Kim D,

Kubzansky LD, Baccarelli A, et al. Psychological factors and DNA methylation of genes related to immune/ inflammatory system markers: the VA Normative Aging Study. BMJ Open 2016;6:e009790. doi:10.1136/bmjopen-2015009790

\section{- Prepublication history and additional material is available. To view please visit the journal (http://dx.doi.org/ 10.1136/bmjopen-2015- 009790).}

Received 21 August 2015 Revised 29 October 2015 Accepted 20 November 2015

CrossMark

For numbered affiliations see end of article.

Correspondence to

Dr Daniel Kim;

dkim@neu.edu

\section{ABSTRACT}

Objectives: Although psychological factors have been associated with chronic diseases such as coronary heart disease (CHD), the underlying pathways for these associations have yet to be elucidated. DNA methylation has been posited as a mechanism linking psychological factors to CHD risk. In a cohort of community-dwelling elderly men, we explored the associations between positive and negative psychological factors with DNA methylation in promoter regions of multiple genes involved in immune/inflammatory processes related to atherosclerosis.

Design: Prospective cohort study.

Setting: Greater Boston, Massachusetts area.

Participants: Samples of 538 to 669 men participating in the Normative Aging Study cohort with psychological measures and DNA methylation measures, collected on 1-4 visits between 1999 and 2006 (mean age $=72.7$ years at first visit).

Outcome measures: We examined anxiety, depression, hostility and life satisfaction as predictors of leucocyte gene-specific DNA methylation. We estimated repeated measures linear mixed models, controlling for age, smoking, education, history of heart disease, stroke or diabetes, \% lymphocytes, $\%$ monocytes and plasma folate.

Results: Psychological distress measured by anxiety, depression and hostility was positively associated, and happiness and life satisfaction were inversely associated with average Intercellular Adhesion Molecule-1 (ICAM-1) and coagulation factor III (F3) promoter methylation levels. There was some evidence that hostility was positively associated with toll-like receptor $2(T L R-2)$ promoter methylation, and that life satisfaction was inversely associated with $T L R-2$ and inducible nitric oxide synthase (iNOS) promoter methylation. We observed less consistent and significant associations between psychological factors and average methylation for promoters of the genes for glucocorticoid receptor (NR3C1), interferon- $\gamma($ IFN- $\gamma)$ and interleukin $6(I L-6)$.

Conclusions: These findings suggest that positive and negative psychological factors affect DNA

\section{Strengths and limitations of this study}

- Strengths of our study include its novel examination of multiple psychological factors (both positive and negative) in relation to DNA methylation in promoter regions of multiple genes plausibly involved in chronic immune/inflammatory processes and inflammation-related endothelial dysfunction.

- We also used repeated measures, thereby improving precision of our estimates.

- A subset of CpG sites was examined for DNA methylation within a gene promoter region; the CpGs from these sites may not necessarily have been good proxies for all $\mathrm{CpGs}$ within the same region.

- The study sample was limited to an elderly, primarily white male population and associations of psychological factors with DNA methylation may be more salient in other population subgroups.

methylation of selected genes involved in chronic immune/inflammatory processes and inflammationrelated endothelial dysfunction. Such epigenetic changes may represent biological pathways that mediate the effects of psychological factors on $\mathrm{CHD}$.

\section{INTRODUCTION}

Although psychological factors and clinical disorders such as anxiety and depression have been linked to a wide variety of health and disease end points including coronary heart disease (CHD) in epidemiological studies, ${ }^{1-3}$ the mechanisms that underlie the associations with CHD have yet to be fully elucidated. CHD has been increasingly characterised as a chronic inflammatory process involving such factors as intercellular adhesion molecules (ie, Intercellular Adhesion 
Molecule-1 (ICAM-1), Vascular Cell Adhesion Molecule-1 (VCAM-1)) facilitating the transendothelial migration of inflammation-related cells into vascular tissues. $^{4}$

DNA methylation may be an intermediary mechanism by which psychological factors influence CHD risk. DNA methylation is a reversible process corresponding to the addition of methyl groups at the 5' position of cytosine rings in $\mathrm{CpG}$ dinucleotides to produce 5-methyl-cytosine $(5 \mathrm{mC})$. DNA methylation is involved in regulation of gene expression and in several genes, lower methylation has been associated with increased mRNA expression. ${ }^{5}$ These relatively stable epigenetic marks can modify gene expression for proteins shaping cellular signals, responses and function. Such modifications may underlie the pathogenesis of major chronic diseases including CHD and cancer. ${ }^{6-8}$ In humans, lower levels of blood LINE-1 DNA methylation have predicted higher risks of cardiovascular diseases, ${ }^{9}$ and alterations in the DNA methylation of specific genes have been linked to higher risks of CHD and cancer. ${ }^{10} 11$

Recent experimental and epidemiological evidence suggests that social/psychological exposures may contribute to the methylation of selected genes/promoters, and may thereby influence gene expression relevant to disease risk factors. ${ }^{5}{ }^{12-17}$ In rats, Weaver et a $\bar{\varphi}$ found that low levels of maternal licking and grooming led to higher cytosine methylation in a glucocorticoid receptor (NR3C1) promoter region in the brain hippocampus of offspring. Such hypermethylation is linked to lower GR expression. Since NR3C1 up-regulation induces negative feedback in the hypothalamic-pituitary-adrenal (HPA) axis, ${ }^{18} 19$ its hypothesised downregulation with negative psychological exposures would potentially generate proinflammatory stress responses. In humans, one study has reported associations between higher anxiety and depressive symptom scores in prenatal women and higher methylation of the NR3C1 gene in newborn cord blood leucocytes and maternal blood leucocytes. ${ }^{12} \mathrm{~A}$ study of younger to middle-aged adults found correlations between a history of childhood adversity with higher leucocyte NR3C1 gene promoter methylation, although no correlations for anxiety and limited correlations for depression with NR3C1 promoter methylation were found. ${ }^{20}$ Distinct methylation patterns have been further observed in depressed versus not depressed individuals, ${ }^{13}$ and lower job seniority has been linked to higher global (Alu line) methylation and methylation in interferon- $\gamma$ (IFN- $\gamma$ ) promoter regions. ${ }^{14}$ Furthermore, individuals of low socioeconomic status (SES) in early life with mothers who expressed high warmth toward them were shown to exhibit less Toll-like receptor (TLR)-stimulated production of interleukin-6 (IL-6) ${ }^{21}$; IL-6 is an inflammatory marker that is predicted by psychosocial factors such as anxiety and depression, and is thought to be involved in the pathogenesis of cardiovascular disease. ${ }^{22}$ Overall, these studies suggest that aspects of the social environment and mood disorders, including anxiety and depression, may induce epigenetic effects. ${ }^{23}{ }^{24}$ Plausibly, these epigenetic changes represent underlying common biological (eg, immune, neuroendocrine) pathways for the putative effects of psychological factors on chronic diseases including CHD.

In a cohort of community-dwelling elderly men in the USA, we explored the associations between positive and negative psychological factors, and DNA methylation in promoter regions of multiple genes involved in chronic immune/inflammatory processes and inflammationrelated endothelial dysfunction. These genes include those for the proteins noted above and for $F_{3}$ (also known as Tissue Factor) and $i N O S$, which have been shown to be involved in chronic inflammatory pathways and have been previously linked to chronic inflammatory conditions. ${ }^{25-30}$

To the best of our knowledge, this is the first study to examine a comprehensive set of psychological factors in relation to epigenetic processes plausibly related to CHD.

\section{MATERIALS AND METHODS}

\section{Study population}

The Normative Aging Study (NAS) is a longitudinal study of ageing established by the US Veterans Administration. The original cohort was recruited between 1961 and 1970, and consisted of 2280 community-dwelling men, aged 21-80 years, from the greater Boston, Massachusetts area, who were free of known chronic medical conditions at enrolment. ${ }^{31}$ Study participants have, every 3 to 5 years, undergone routine physical examinations and laboratory tests, and responded to surveys on medical history, and lifestyle and psychological factors.

The present study analysed data on men participating in the NAS cohort, with psychological measures and DNA methylation measures (average of 2.2 measures/ individual), collected on between one to four visits between 1999 and 2006. During this period, 765 study participants provided at least one whole blood sample that was used to measure DNA methylation. Since for some participants the extracted DNA was not sufficient in quantity to conduct methylation assays for all genes, and due to some assay failures, the total numbers of men in whom there were assays corresponding to promoter regions of different genes varied..$^{32}$

\section{Outcome variables}

The average and position-specific levels of methylation in promoter regions of seven genes (toll-like receptor 2 (TLR-2), coagulation factor III (F3), glucocorticoid receptor (NR3C1), intercellular adhesion molecule-1 (ICAM-1), interferon- $\gamma(I F N-\gamma)$, interleukin 6 (IL- $\sigma)$ and inducible nitric oxide synthase (iNOS)) were analysed as outcomes in separate models.

These genes were selected based on past evidence for associations of: (1) proteins coded by these genes in 
animal and/or human studies of atherosclerosis or the pathophysiology of heart disease; (2) psychological factors with methylation of promoters of the genes; (3) psychological factors with peripheral blood levels of the markers expressed by these genes. For instance, for the first selection criterion, both serum ICAM- 1 and $I L-6$ levels have independently predicted CHD risk in prospective studies after controlling for demographic/socioeconomic and traditional CHD risk factors. ${ }^{33}{ }^{34}$ In the Introduction, we cited studies suggesting linkages between psychological exposures and the methylation of NR3C1 and IFN- $\gamma$ promoters, which in turn might explain chronic inflammatory processes characterising diseases such as CHD. As an example for the third selection criterion, lower early-life socioeconomic status (SES) has been linked to greater expression of both NR3C1 and TLR receptor mRNA in leucocytes. ${ }^{35}$

DNA was extracted from a stored frozen buffy coat of $7 \mathrm{~mL}$ whole blood, using the QiAmp DNA blood kits (QIAGEN, Hilden, Germany); 500 ng DNA (concentration $50 \mathrm{ng} / \mu \mathrm{L}$ ) was treated using EZ DNA MethylationGold Kit (Zymo Research, Orange, California, USA) according to the manufacturer's protocol. Final elution was performed with $30 \mu \mathrm{L}$ of M-Elution Buffer.

CpG dinucleotide-rich promoter regions were identified using the Genomatix Software Suite (Genomatix, Germany). Promoters without any assigned transcripts were excluded. To the best of our knowledge, there were no DNA methylation assays for the genes analysed that were already published. Therefore, we developed new pyrosequencing assays by selecting amplicons in promoter CpG-rich areas. For each gene, the PCR-pyrosequencing primer (more than 20 base pairs long) of the highest available quality that was associated with one of the promoters was designed using specialised software (PSQ Assay Design, Biotage, Sweden). The fractions of $\mathrm{CpG}$ sites examined by gene were as follows: TLR-2 (5/49); F3 (5/78); NR3C1 (1/7); ICAM-1 (5/69); IFN- $\gamma$ (2/8); IL-6 (2/18); iNOS (2/8). We did not assay higher proportions of CpG sites due to inherent limitations of the method applied, that is, we excluded PCR amplicons with 350 base pairs or longer, primers that avoided CpGs and target sequences of 40 base pairs or longer, to optimise PCR and sequencing conditions. Online supplementary table S1 lists the specific CpG positions for DNA methylation that we measured within specified promoter regions for each gene. We had limited information about the CpGs that were analysed (eg, for NR3C1), including their functionality or their proximity to transcription factor-binding sites or other important sequences. Since genomic locations were for the hg18 genome build, the majority of the CpGs that we examined were not assayed by the most common methylation assays (ie, either the $27 \mathrm{~K}$ or $450 \mathrm{~K}$ assays) that are available in public data sets.

The degree of methylation was calculated as the percentage of methylated cytosine residues divided by the sum of methylated and unmethylated cytosine residues
$(\% 5 \mathrm{mC})$ in each sample. Built-in controls were used to verify bisulfite conversion efficiency. Each sample was tested twice for each marker to improve statistical power and precision. The average of the replicates was used.

\section{Predictor variables}

We used data on anxiety and depression measured through the Brief Symptom Inventory (BSI), a selfadministered 53-item questionnaire of nine primary psychological symptom dimensions (anxiety, depression, hostility, interpersonal sensitivity, obsessive-compulsive, paranoid ideation, phobic anxiety, psychoticism, somatisation) experienced by the respondent over the previous 30 days; the BSI was included as part of the Health and Social Behavior Survey in the NAS, starting in $1985 .{ }^{31}{ }^{36}$ Happiness (based on the single item 'How happy are you right now?') and life satisfaction (based on the 11-item version of the Life Satisfaction Inventory-A) ${ }^{37}$ were also examined as predictor variables. Higher life satisfaction scale scores corresponded to higher self-reported life satisfaction; higher scores on the other scales reflected higher negative psychological symptoms. All psychological measures were analysed as continuous. Internal consistency reliability (Cronbach's $\alpha$ ) values for the anxiety, depression, hostility and life satisfaction scales were all acceptably high $(>0.70)$.

\section{Covariates}

Model covariates consisted of the age at first visit in or after 1999 (years), smoking (pack-years of smoking), education (>high school, $\leq$ high school), history of CHD or stroke prior to 1999, history of diabetes prior to 1999, $\%$ basophils, \% eosinophils, \% lymphocytes, \% monocytes, \% neutrophils and plasma folate levels. Previous evidence suggests that leucocyte composition is related to DNA methylation, ${ }^{38}$ and that folate is a source of methyl groups and folate depletion leads to lower blood DNA methylation. ${ }^{39}$ Since $98 \%$ of the sample was white, we did not adjust for race/ethnicity. In sensitivity analyses, we additionally controlled for baseline hypertension (ie, hypertension prior to 1999) and total serum cholesterol.

\section{Statistical analysis}

We first calculated descriptive statistics (mean, range, percentages for psychological factors and covariates, mean percentage methylation for gene-specific promoter methylation) based on study participants with measures of ICAM-1 promoter region methylation, which showed several significant associations.

We then constructed a Pearson correlation coefficient matrix for the psychological factors and a correlation coefficient matrix for the methylation outcomes.

To examine the associations between the psychological factors and the methylation outcomes, we next estimated repeated measures linear mixed models (equivalent to random intercept models) to account for up to four repeated measures, using a first-order autoregressive 
covariance structure (in which a decreasing correlation of SEs over time was modelled). The log-likelihood fit statistics for the models indicated better model fits than those for the corresponding models using a compound symmetry covariance structure; unstructured covariance structure models did not converge. Since we assumed a short latency period for methylation changes, ${ }^{40-43}$ we modelled each psychological factor as a predictor of gene-specific methylation measured on the same visit (averaged across cytosines in CpG sites within the promoter region, varying from one $\mathrm{CpG}$ site for the NR3C1 gene to five CpG sites for the $F 3$ gene according to the density of CpG sites in the sequence amplified within the promoter region). In addition, we noted the associations between selected covariates (age, smoking, income/education) and methylation.

For primary associations significant at the $5 \%$ level, we further tested for dose-response relationships, by grouping the respective psychological factor into meaningful and/or equally-sized categories where possible. A doseresponse relationship would lend support to a casual association. ${ }^{44} \mathrm{~A}$ linear test for trend was performed by converting the categories into an ordinal variable and noting its corresponding $\mathrm{p}$ value.

We further examined the associations between psychological factors and serum ICAM-1, to examine whether similar relationships were present as between the psychological factors and ICAM-1 promoter methylation levels (because the latter would be expected to be inversely related to ICAM-1 expression).

Finally, because of the known association between ageing and methylation, we repeated the analyses using age $^{2}$ as an additional covariate to saturate the model for an age effect, and found comparable results (data not shown). Additional sensitivity analyses explored the robustness of the findings after controlling for household income, baseline hypertension and total serum cholesterol.

All tests were two-tailed with a 5\% significance level. All analyses were conducted using SAS V.9.1 (SAS Institute, Cary, North Carolina, USA).

All participants gave written informed consent. This research was approved by the human subjects committees of the Boston VA Medical Center and the Harvard School of Public Health.

\section{RESULTS}

\section{Characteristics of study sample}

Table 1 shows descriptive characteristics of the study sample based on 616 men with measures of ICAM-1 promoter region methylation. We present characteristics for this sample because several of the corresponding associations with ICAM-1 methylation were significant among the different gene promoter regions analysed. The sample had a mean age of 72.5 years (range 56100 years) at first visit. Approximately one-third (34.1\%) attained no more than high school education and over
Table 1 Descriptive statistics (mean values with ranges in parentheses; percentages) for samples analysed with respective characteristic and ICAM-1 promoter methylation (n ranging from 538 to 577 men)

\begin{tabular}{lc} 
Mean age in years at first visit in 1999 & $72.5(56-100)$ \\
Percentage $\leq$ high school & 34.1 \\
Percentage white & 98.0 \\
Percentage with CHD/stroke/diabetes & 33.3 \\
before 1999 & \\
Smoking in pack-years & $21.8(0-131)$ \\
Anxiety & $0.2(0-2.83)$ \\
Depression & $0.2(0-3.33)$ \\
Hostility & $0.2(0-3.00)$ \\
Happiness & $7.4(1-9)$ \\
Life satisfaction & $7.9(0-11)$ \\
Percentage basophils & $0.6(0-2)$ \\
Percentage eosinophils & $3.2(0-22)$ \\
Percentage lymphocytes & $26.0(5-90)$ \\
Percentage monocytes & $8.8(0-17)$ \\
Percentage neutrophils & $61.65(3-85)$ \\
Plasma folate (ng/mL) & $17.41(3.3-99.3)$ \\
DNA methylation in gene promoter regions $(\%)$ \\
TLR-2 & $3.1(0-8.9)$ \\
F3 & $2.3(0-14.8)$ \\
NR3C1 & $47.0(14.7-72.8)$ \\
ICAM-1 & $4.4(1.7-16.1)$ \\
IFN- $\gamma$ & $84.4(30.9-95.7)$ \\
IL-6 & $43.7(10.3-86.6)$ \\
iNOS & $69.7(24.5-87.2)$ \\
\hline CHD, coronary heart disease; F3, coagulation & \\
&
\end{tabular}

CHD, coronary heart disease; F3, coagulation factor III; ICAM-1, intercellular adhesion molecule-1; IFN- $\gamma$, interferon- $\gamma$; IL-6, interleukin 6; iNOS, inducible nitric oxide synthase; NR3C1, glucocorticoid receptor; TLR-2, toll-like receptor 2 .

two-thirds had previously smoked, with an average of 21.8 pack-years of smoking (table 1 ). These characteristics were similar to those of the larger cohort of men with visits between 1999 and 2006, including men with missing observations for methylation $(\mathrm{n}=1121$ men: mean age 71.7 years, \% with less than high school education $=35.9$; mean pack-years of smoking=21.6). After listwise deletion of missing data in respective models, the sizes of analytic samples ranged from 481 to 669 men. Missing gene-specific methylation data ranged from $5.4 \%(I F N-\gamma)$ to $23.8 \%$ (iNOS), due to the presence of assay failures and the lack of sufficient DNA, which disproportionately affected genes that were tested later in the order (ie, iNOS, ICAM-1). Missing model covariate data ranged collectively from $3.1 \%$ to $3.5 \%$. Missing psychological factor data ranged from $3.7 \%$ (happiness) to $10.8 \%$ (life satisfaction) in the respective model (see online supplementary table S2). Mean leucocyte methylation levels within promoter regions ranged from 2.2\% $5 \mathrm{mC}$ (OGG gene) to $84.8 \% 5 \mathrm{mC}$ (IFN- $\gamma$ gene); none of the distributions was highly skewed (table 1). Intraindividual changes in leucocyte methylation ranged from 1.4 to 2.4 times the SD across repeated measures.

Anxiety, depression and hostility scale scores were significantly positively correlated with one another, and 
were nearly all significantly inversely correlated with happiness and life satisfaction scores (all $|\mathrm{r}|>0.3$ and $\mathrm{p}<0.01$; table 2). By contrast, none of the methylation outcomes were moderately to strongly correlated with one another (all $|\mathrm{r}|<0.3$; data not shown), suggesting that these outcomes represented relatively independent events and processes.

\section{Associations between psychological factors and average DNA methylation}

Table 3 shows the multivariate-adjusted coefficient estimates from repeated measures models. Negative psychological factors were related to higher average methylation in ICAM-1 promoter regions (with the associations for anxiety significant at the 0.10 level and for depression significant at the 0.05 level). Happiness was significantly inversely associated with ICAM-1 promoter methylation. Depression was significantly positively associated, and happiness and life satisfaction were significantly inversely associated, with average methylation in F3 promoter regions, respectively. For TLR-2 promoter methylation, all negative psychological factors showed positive relations (with the association for hostility significant at the 0.10 level), and both positive psychological factors showed inverse relations (with the association for life satisfaction significant at the 0.05 level). For iNOS promoter methylation, all negative psychological factors showed inverse relations and both positive psychological factors showed positive relations. However, only the association for life satisfaction was significant at the 0.10 level. For NR3C1 promoter methylation, depression, hostility, happiness and life satisfaction, all exhibited positive and non-significant associations. Likewise, psychological factors were inconsistently and non-significantly related to higher methylation in the promoter regions for $I F N-\gamma$ and $I L-6$.

For all associations significant at the 0.05 level, we further identified monotonic dose-response relationships, with categories of higher scores of the psychological factors being associated with stronger associations. Tables 4 and 5 show the coefficient estimates across categories as well as the $p$ values from the tests for linear trend across categories; these $\mathrm{p}$ values were significant at the 0.05 level for $F^{3}$ promoter methylation and at the 0.10 level for ICAM-1 promoter region methylation, respectively.
In all models, pack-years of smoking significantly predicted higher average methylation levels in the genespecific promoter regions. Age was non-significantly inversely associated with methylation. Additional adjustment for household income (with lower income being non-significantly positively associated with methylation), baseline hypertension and total serum cholesterol, did not alter the main results (data not shown).

\section{Associations between psychological factors and serum ICAM-1}

No psychological factors were associated with serum ICAM-1 levels (for anxiety: $\beta=5.11, p=0.51$; other psychological factors exhibited similar associations). ICAM-1 methylation levels and serum ICAM-1 levels were uncorrelated $(\mathrm{r}=-0.04)$.

\section{DISCUSSION}

In this study of community-dwelling elderly adult men, we found consistent associations between both, positive and negative psychological factors, with higher average leucocyte DNA methylation in ICAM- 1 promoter regions and in $\mathrm{F}_{3}$ promoter regions. There was some evidence that hostility was positively associated with TLR-2 promoter methylation, and that life satisfaction was inversely associated with both, TLR-2 and $i N O S$ promoter methylation. We observed less consistent and significant associations between psychological factors and average methylation for promoters of the genes for NR3C1, $I F N-\gamma$ and $I L-6$.

Our main findings were generally robust across multiple BSI component scales. While this may stem from similarities across component scale measures, results using very different scales (eg, life satisfaction) were qualitatively consistent. Moreover, smoking has been linked to proinflammatory states and atherosclerosis, ${ }^{45}$ and the direction of the associations for smoking with hypermethylation of ICAM-1 promoter regions matched those for negative psychological factors, providing support that the associations were not simply attributable to chance. Our findings were, furthermore, robust to the adjustment of the presence of CHD, stroke and diabetes, countering underlying comorbidities/health selection as alternative explanations for the main findings.

Table 2 Pearson correlation coefficients between psychological factors ${ }^{*}$

\begin{tabular}{|c|c|c|c|c|c|}
\hline & Anxiety & Depression & Hostility & Happiness & Life satisfaction \\
\hline Anxiety & 1.00 & $0.76(n=611)$ & $0.67(n=611)$ & $-0.32(n=612)$ & $-0.31(n=578)$ \\
\hline Depression & & 1.00 & $0.63(n=609)$ & $-0.46(n=611)$ & $-0.42(n=577)$ \\
\hline Hostility & & & 1.00 & $-0.30(n=610)$ & $-0.28(n=577)$ \\
\hline Happiness & & & & 1.00 & $0.58(n=598)$ \\
\hline Life satisfaction & & & & & 1.00 \\
\hline
\end{tabular}

${ }^{*}$ For men with observations for the pair of psychological factors.

$\mathrm{p}<0.01$ for all correlations. 


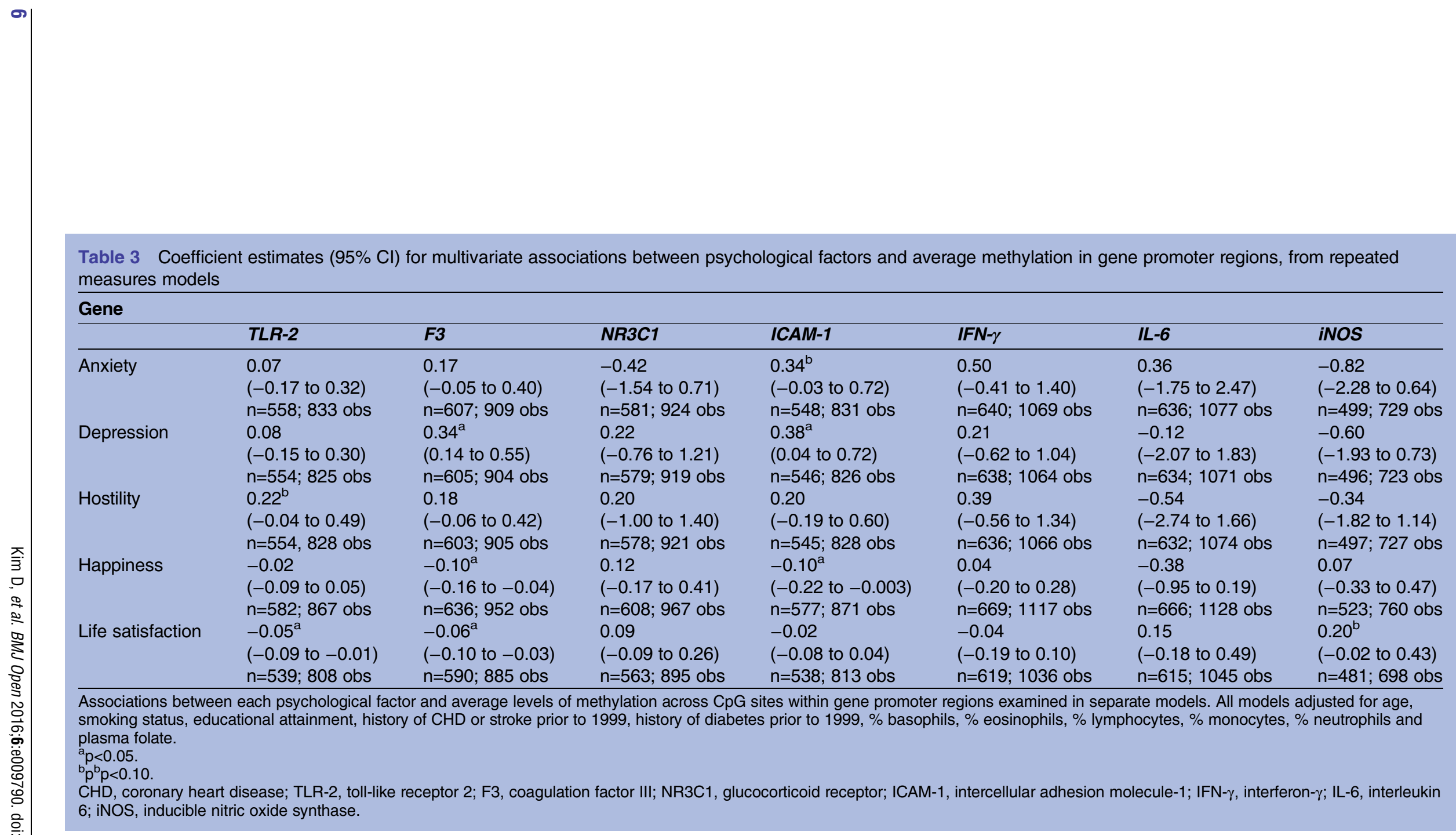


Table 4 Coefficient estimates from repeated measures models for multivariate associations between categorised scale values of depression, happiness, life satisfaction and F3 promoter methylation ( $\mathrm{n}=658$ men, 988 observations)

\begin{tabular}{|c|c|c|c|}
\hline & $\begin{array}{l}\text { Coefficient } \\
\text { estimate }\end{array}$ & $95 \% \mathrm{Cl}$ & p Value \\
\hline \multicolumn{4}{|l|}{ Depression } \\
\hline 0 & - & - & - \\
\hline $0.01-0.4$ & -0.13 & -0.34 to 0.09 & 0.24 \\
\hline$>0.4$ & 0.33 & 0.10 to 0.56 & $\begin{array}{l}0.005 \\
P_{\text {trend }}=0.03\end{array}$ \\
\hline \multicolumn{4}{|l|}{ Happiness } \\
\hline $\begin{array}{l}1-4 \\
\text { (unhappy) }\end{array}$ & - & - & \\
\hline $5-7$ & -0.20 & -0.54 to 0.14 & 0.24 \\
\hline \multicolumn{4}{|l|}{ (happy) } \\
\hline & & & $\mathrm{P}_{\text {trend }}<0.001$ \\
\hline \multicolumn{4}{|c|}{ Life satisfaction } \\
\hline $0-5$ & - & - & - \\
\hline $6-8$ & -0.28 & -0.49 to -0.06 & 0.01 \\
\hline $9-11$ & -0.40 & -0.60 to -0.20 & $\begin{array}{l}<0.001 \\
\mathrm{P}_{\text {trend }}<0.001\end{array}$ \\
\hline \multicolumn{4}{|c|}{$\begin{array}{l}\text { F3 methylation values corresponded to the average levels of } \\
\text { methylation across CpG sites within the F3 promoter region. } \\
\text { All models adjusted for age, smoking status, educational } \\
\text { attainment, history of CHD or stroke prior to } 1999, \text { history of } \\
\text { diabetes prior to } 1999, \% \text { basophils, \% eosinophils, \% } \\
\text { lymphocytes, \% monocytes, \% neutrophils and plasma folate. } \\
\text { CHD, coronary heart disease; F3, coagulation factor III. }\end{array}$} \\
\hline
\end{tabular}

Higher circulating levels of serum ICAM-1 have been previously independently linked to modest risks of CHD after adjusting for key covariates such as SES. ${ }^{4} 46 \quad 47$

Table 5 Coefficient estimates from repeated measures models for multivariate associations between categorised scale values of depression and happiness, and ICAM-1 promoter methylation ( $\mathrm{n}=600$ men, 906 observations)

\begin{tabular}{|c|c|c|c|}
\hline & $\begin{array}{l}\text { Coefficient } \\
\text { estimate }\end{array}$ & $95 \% \mathrm{Cl}$ & p Value \\
\hline \multicolumn{4}{|l|}{ Depression } \\
\hline 0 & - & - & - \\
\hline $0.01-0.4$ & 0.19 & -0.16 to 0.55 & 0.29 \\
\hline$>0.4$ & 0.30 & -0.09 to 0.70 & $\begin{array}{l}0.13 \\
P_{\text {trend }}=0.09\end{array}$ \\
\hline \multicolumn{4}{|l|}{ Happiness } \\
\hline $\begin{array}{l}\text { 1-4 (not } \\
\text { happy) }\end{array}$ & - & - & - \\
\hline $5-7$ & -0.21 & -0.76 to 0.34 & 0.46 \\
\hline 8-9 (happy) & -0.42 & -0.97 to 0.13 & $\begin{array}{l}0.13 \\
P_{\text {trend }}=0.06\end{array}$ \\
\hline
\end{tabular}

ICAM-1 methylation values corresponded to the average levels of methylation across $\mathrm{CpG}$ sites within the ICAM-1 promoter region. All models adjusted for age, smoking status, educational attainment, history of CHD or stroke prior to 1999 , history of diabetes prior to $1999, \%$ basophils, \% eosinophils, \% lymphocytes, \% monocytes, \% neutrophils and plasma folate. $\mathrm{CHD}$, coronary heart disease; ICAM-1, intercellular adhesion molecule-1.
Notably, we found no association between psychological factors and serum ICAM-1. Along with the presence of associations between psychological factors and ICAM-1 promoter methylation, this could be explained by the fact that serum ICAM- 1 is derived from multiple sources (vascular endothelium, macrophages, lymphocytes), consistent with the absence of a correlation between leucocyte ICAM-1 methylation and serum ICAM-1. Past investigations of the NAS have likewise found no association between serum ICAM-1 and LINE-1 leucocyte methylation levels. ${ }^{48}$ Whether methylation of ICAM-1 in white cell count predicts serum ICAM-1 levels derived solely from white cell count (vs other sources), and whether this ICAM-1 independently contributes to higher risks of CHD, should be explored in future studies.

Atherosclerosis is a chronic inflammatory process involving the infiltration of leucocytes into the extravascular space, mediated in part by adhesion molecules. Smooth muscle cells participate in this process by expressing adhesion molecules such as VCAM- 1 and ICAM- $1 .{ }^{49}$ ICAM- 1 plays a pivotal role in the adhesion of leucocytes to the endothelium. ${ }^{50-52}$ Given evidence that psychological factors are risk factors for atherosclerosis, ${ }^{1}$ one possible explanation for negative psychological factors being linked to higher ICAM-1 promoter region methylation in leucocytes is cellular signalling, with ICAM-1 being known to function via signal transduction $^{53} 54$ Low binding of leucocyte ICAM-1 to its cell membrane integrins could trigger a cascade of proinflammatory mediators and signal endothelial cells to release ICAM- $1,,^{52} 55-57$ and could thereby stimulate ICAM-1 leucocyte binding to vascular endothelial cells. Hence, through signalling mechanisms, low leucocyte ICAM-1 levels could induce leucocyte migration into vascular endothelial tissues. Future biological studies (eg, animal experiments that manipulate distress or other exposures) should further investigate and test this and other potential pathways.

Depression was positively associated, and happiness and life satisfaction were each inversely associated, with higher $F 3$ promoter methylation in leucocytes (which in turn would be linked to reduced leucocyte F3 expression). Some evidence suggests that the major source of $F 3$ in arterial thrombosis is the vascular wall rather than monocytes, ${ }^{25}$ although monocyte $F 3$ also contributes to inflammation and thrombosis. F3, also known as Tissue Factor, has been shown to be involved in cellular signalling and inflammatory pathways. ${ }^{26} 27$ Similar to the hypothesis for $I C A M-1$, low leucocyte $F 3$ levels via signalling pathways may promote inflammatory states through greater vascular $F 3$ levels.

Furthermore, hostility was positively associated and life satisfaction was inversely associated with higher $T L R-2$ promoter methylation, which would imply lower $T L R-2$ expression. These findings appear contrary to the hypothesised role that $T L R-2$ plays in atherosclerosis. ${ }^{28} 29$ Nonetheless, there is some evidence to suggest that 
$T L R-2$ promoter hypermethylation is present in chronic inflammatory processes such as periodontitis. ${ }^{30}$ In addition, it has been suggested that the inflammatory process itself may induce cytosine damage and aberrant methylation patterns, including hypermethylation. ${ }^{58}$ Furthermore, the association of negative psychological states such as hostility with decreased expression of TLR-2 may signify suppression of the immune system; this is consistent with observed relationships between stress and immune suppression in other studies. ${ }^{59}$

We found no associations between psychological factors and leucocyte NR3C1 promoter methylation. Previous studies in humans have yielded conflicting results. For example, an investigation in prenatal women, using clinically-administered (Hamilton Rating) scales of anxiety and depression and a self-administered (Edinburgh Postnatal Depression) scale of depression, observed associations between higher maternal anxiety, and depressive symptom scores and methylation of CpGs within the promoter and exon $1 \mathrm{~F}$ of the NR3C1 gene (homologous to the $1_{7}$ region of the rat NR3C1 gene) in maternal blood leucocytes. ${ }^{12}$ A study of men and women aged 18-59 years reported correlations between a history of childhood adversity with higher leucocyte NR3C1 gene promoter methylation, yet found no correlations for anxiety (using the State-Trait Anxiety Inventory) and only limited correlations for depression (using the Inventory for Depressive Symptoms) with GR promoter methylation (at 0 of $13 \mathrm{CpG}$ sites and 2 of $13 \mathrm{CpG}$ sites, respectively).$^{20}$ Meanwhile, a recent brain postmortem study in adults found no hippocampal GR promoter methylation differences between those clinically diagnosed with major depression versus controls. ${ }^{60}$

Strengths of our study include its examination of multiple psychological factors (both positive and negative) and its novel exploration of DNA methylation in promoter regions of multiple genes plausibly involved in chronic immune/inflammatory processes and inflammation-related endothelial dysfunction; and its reliance on a community-based sample, which strengthens the generalisability of our findings. We further tested for and confirmed linear dose-response relationships, which supports the presence of causal associations.

There were several limitations to our study. First, we examined DNA methylation at a subset of CpG sites within a gene promoter region. The inability to assay high proportions, given methodological limitations, could have led us to the omission of some relevant CpG sites. The analysed CpGs (selected based on the aforementioned methodological limitations) may not necessarily have been good proxies for the rest of the CpGs within the same regions. Second, differences in results from previous studies, particularly for NR3C1 methylation, might also stem from the measurement of methylation in peripheral blood rather than hippocampal tissue; methylation effects may be tissue-specific. ${ }^{20} 61$ Third, due to the multiple associations examined, the multiple comparisons problem, whereby multiple comparisons may increase the presence of significant associations by chance, cannot be ruled out. Fourth, while the null associations for methylation in promoter regions of several genes including $N R 3 C 1, I F N-\gamma$ and $I L-6$, could reflect the true absence of associations, they could also possibly be attributed to selection bias due to attrition or missing methylation data, as suggested by demographic (age, education) differences in those analysed versus the NAS cohort in 1985, when the BSI was first administered. For instance, those with a stronger association between the psychological factors and methylation may have either died or been lost to follow-up, leading to attenuated and null associations in the analysed data. With respect to the varying sample sizes between analytic samples for genes examined, the mechanism of missing data due to insufficient DNA and assay failures was plausibly missing completely at random (MCAR), and entirely unrelated to the levels of methylation of a particular sequence of DNA. ${ }^{32}$ Under the MCAR mechanism, the listwise deletion method that we applied should be valid. ${ }^{62}$ In support of the MCAR assumption being met, we determined that those participants with and without missing methylation data for each gene were generally comparable on demographic characteristics (mean age, distribution of education), mean pack-years of smoking, and mean anxiety and depression scores. Fifth, the NAS cohort does not currently have genome-wide association study (GWAS) data. Hence, we could not specifically evaluate the interplay between genetics and DNA methylation and further studies are warranted. Sixth, we lacked measures of additional cell subtypes (eg, B cells, $\mathrm{T}$ cells and natural killer cells, as subtypes of lymphocytes), which may have biased our results through residual confounding. Finally, the presence of null associations may in part be due to the study sample being limited to an elderly, primarily white male population. Effects of psychological factors on DNA methylation may be more salient in other population subgroups, or at earlier, sensitive time points over the life course. Future studies should extend examination of these associations to younger adults, older women and members of other racial/ethnic groups.

In summary, our study primarily suggests novel relations between positive and negative psychological factors, and methylation of ICAM-1 promoter regions and linkages with F3 gene methylation, and, to a lesser extent, associations with TLR-2 promoter methylation. Confirming these findings in other populations and settings may yield a better understanding of the epigenetic mechanisms by which psychological factors influence CHD and other major chronic disease outcomes.

\section{Author affiliations}

${ }^{1}$ Department of Health Sciences, Northeastern University, Boston, Massachusetts, USA

${ }^{2}$ Department of Social and Behavioral Sciences, Harvard School of Public Health, Boston, Massachusetts, USA

${ }^{3}$ Department of Human and Social Sciences, EHESP French School of Public Health, Rennes, France 
${ }^{4}$ Department of Environmental Health, Harvard School of Public Health, Boston, Massachusetts, USA

${ }^{5}$ Boston University School of Medicine, Boston, Massachusetts, USA

${ }^{6}$ VA Normative Aging Study, VA Boston Healthcare System, Boston,

Massachusetts, USA

${ }^{7}$ Department of Epidemiology, Boston University School of Public Health, Boston, Massachusetts, USA

${ }^{8}$ Department of Psychiatry, Boston University School of Medicine, Boston, Massachusetts, USA

${ }^{9}$ University of Milan, Milan, Italy

${ }^{10}$ IRCCS Maggiore Policlinico Hospital, Milan, Italy

${ }^{11}$ Department of Epidemiology, Harvard School of Public Health, Boston, Massachusetts, USA

Acknowledgements The authors thank John Hutchinson from the Harvard TH Chan School of Public Health for technical assistance.

Contributors DK, LDK and JS conceived and designed the study. AB, DS, AS LT, LC, PV and JS gathered data. DK performed all data analyses and drafted the manuscript. DK, LDK, AB, LT and JS revised the manuscript for important intellectual content.

Funding This research was supported by the National Institutes of Health (ES05257-06A1, ES014663, ES15172, ES00002, P20-MD000501, R01 ES07821, P42-ES05947, R01-AG02237, R29-AG07465, R01-AG08436) and the National Center for Research Resources General Clinical Research Centers programme (M01RR02635); by the US Environmental Protection Agency (R832416); by the Cooperative Studies Programme/ERIC of the US Department of Veterans Affairs; and is a component of the Massachusetts Veterans Epidemiology Research and Information Center (MAVERIC); and by a VA Research Career Scientist award to David Sparrow. Daniel Kim was supported by a career development Pathway to Independence Award through the National Heart, Lung, and Blood Institute of the National Institutes of Health (grant R0O HL089459); Andrea Baccarelli is supported by National Institute of Environmental Health Sciences grant ES000002.

Competing interests None declared.

Ethics approval Boston VA Medical Center, Harvard School of Public Health.

Provenance and peer review Not commissioned; externally peer reviewed.

Data sharing statement Data are from the Normative Aging Study, from which restricted data are available for researchers who meet the criteria.

Open Access This is an Open Access article distributed in accordance with the terms of the Creative Commons Attribution (CC BY 4.0) license, which permits others to distribute, remix, adapt and build upon this work, for commercial use, provided the original work is properly cited. See: http:// creativecommons.org/licenses/by/4.0/

\section{REFERENCES}

1. Kuper $\mathrm{H}$, Marmot $M$, Hemingway $H$. Systematic review of prospective cohort studies of psychosocial factors in the etiology and prognosis of coronary heart disease. Semin Vasc Med 2002;2:267-314.

2. Roest AM, Martens EJ, de Jonge $P$, et al. Anxiety and risk of incident coronary heart disease: a meta-analysis. J Am Coll Cardiol 2010;56:38-46.

3. Gan $Y$, Gong $Y$, Tong $X$, et al. Depression and the risk of coronary heart disease: a meta-analysis of prospective cohort studies. BMC Psychiatry 2014;14:371.

4. Malik I, Danesh J, Whincup P, et al. Soluble adhesion molecules and prediction of coronary heart disease: a prospective study and meta-analysis. Lancet 2001;358:971-6.

5. Weaver ICG, Cervoni N, Champagne FA, et al. Epigenetic programming by maternal behavior. Nat Neurosci 2004;7:847-54.

6. Gluckman PD, Hanson MA, Buklijas T, et al. Epigenetic mechanisms that underpin metabolic and cardiovascular diseases. Nat Rev Endocrinol 2009;5:401-8.

7. Fraga MF, Esteller M. Epigenetics and aging: the targets and the marks. Trends Genet 2007;23:413-18.
8. Turunen MP, Aavik E, Yla-Herttuala S. Epigenetics and atherosclerosis. Biochim Biophys Acta 2009;1790:886-91.

9. Baccarelli A, Wright R, Bollati V, et al. Ischemic heart disease and stroke in relation to blood DNA methylation. Epidemio 2010;21:819-28.

10. Baccarelli A, Rienstra M, Benjamin EJ. Cardiovascular epigenetics: basic concepts and results from animal and human studies. Circ Cardiovasc Genet 2010;3:567-73.

11. Kulis M, Esteller M. DNA methylation and cancer. Adv Genet 2010;70:27-56.

12. Oberlander TF, Weinberg J, Papsdorf M, et al. Prenatal exposure to maternal depression, neonatal methylation of human glucocorticoid receptor gene (NR3C1) and infant cortisol stress responses. Epigenetics 2008;3:97-106

13. Uddin M, Koenen KC, Aiello AE, et al. Epigenetic and inflammatory marker profiles associated with depression in a community-based epidemiologic sample. Psychol Med 2010;14:1-11.

14. Bollati V, Baccarelli A, Sartori S, et al. Epigenetic effects of shiftwork on blood DNA methylation. Chronobiol Int 2010;27:1093-104.

15. Uddin M, Aiello AE, Wildman DE, et al. Epigenetic and immune function profiles associated with posttraumatic stress disorder. Proc Natl Acad Sci USA 2010;107:9470-5.

16. McGowan PO, Sasaki A, D'Alessio AC, et al. Epigenetic regulation of the glucocorticoid receptor in human brain associates with childhood abuse. Nat Neurosci 2009;12:342-8.

17. Beach $\mathrm{SRH}$, Brody $\mathrm{GH}$, Todorov AA, et al. Methylation at 5HTT mediates the impact of child sex abuse on women's antisocial behavior: an examination of the lowa Adoptee sample. Psychosom Med 2011;73:83-7.

18. McQuade R, Young AHY. Future therapeutic targets in mood disorders: the glucocorticoid receptor. $\mathrm{Br} J$ Psychiatry 2000;177:390-5.

19. Yehuda R, Seckl J. Mini-review: stress-related psychiatric disorders with low cortisol levels: a metabolic hypothesis. Endocrinology 2011;152:4496-503.

20. Tyrka AR, Price LH, Marsit C, et al. Childhood adversity and epigenetic modulation of the leukocyte glucocorticoid receptor: preliminary findings in healthy adults. PLOS ONE 2012;7: e30148.

21. Chen E, Miller GE, Kobor MS, et al. Maternal warmth buffers the effects of low early-life socioeconomic status on pro-inflammatory signaling in adulthood. Mol Psychiatry 2011;16:729-37.

22. Morozink JA, Friedman EM, Coe CL, et al. Socioeconomic and psychosocial predictors of interleukin- 6 in the MIDUS national sample. Health Psychol 2010;29:626-35.

23. Szyf M, McGowan P, Meaney MJ. The social environment and the epigenome. Environ Mol Mutagen 2008;49:46-60.

24. McGowan PO, Kato T. Epigenetics in mood disorders. Environ Health Prev Med 2008;13:16-24

25. Day SM, Reeve JL, Pedersen B, et al. Macrovascular thrombosis is driven by tissue factor derived primarily from the blood vessel wall. Blood 2005;105:192-8.

26. Konigsberg W, Kirchhofer D, Riederer MA, et al. The TF:VIIa complex: clinical significance, structure-function relationships and its role in signaling and metastasis. Thromb Haemost 2001;86: 757-71.

27. Lu P, Liu J, Pang X. Pravastatin inhibits fibrinogen- and FDP-induced inflammatory response via reducing the production of IL-6, TNF- $\alpha$ and iNOS in vascular smooth muscle cells. Mol Med Rep 2015;12:6145-51.

28. Curtiss LK, Tobias PS. Emerging role of Toll-like receptors in atherosclerosis. J Lipid Res 2009;50(Suppl):S340-5.

29. Cole JE, Georgiou E, Monaco C. The expression and functions of Toll-like receptors in atherosclerosis. Mediators Inflamm 2010;2010:393946.

30. de Faria Amormino SA, Arao TC, Saraiva AM, et al. Hypermethylation and low transcription of TLR2 gene in chronic periodontitis. Human Immunol 2013;74:1231-6.

31. Rajan P, Kelsey KT, Schwartz JD, et al. Lead burden and psychiatric symptoms and the modifying influence of the delta-aminolevulinic acid dehydratase (ALAD) polymorphism: the VA Normative Aging Study. Am J Epidemiol 2007;166:1400-8.

32. Alexeeff SE, Baccarelli AA, Halonen J, et al. Association between blood pressure and DNA methylation of retrotransposons and pro-inflammatory genes. Int J Epidemiol 2013;42:270-80.

33. Rodondi N, Marques-Vidal P, Butler J, et al. Markers of atherosclerosis and inflammation for prediction of coronary heart disease. Am J Epidemiol 2010;171:540-9.

34. Shai I, Pischon T, Hu FB, et al. Soluble intercellular adhesion molecules, soluble vascular cell adhesion molecules, and risk of coronary heart disease. Obesity 2006;14:2099-106. 
35. Miller G, Chen E. Unfavorable socioeconomic conditions in early life presage expression of proinflammatory phenotype in adolescence. Psychosom Med 2007;69:402-9.

36. Derogatis LR, Melisaratos N. The Brief Symptom Inventory: an introductory report. Psychol Med 1983;13:595-605.

37. Liang J. Dimensions of the life satisfaction index A: a structural formulation. Journal of Gerontology 1984;39:613-22.

38. Lam LL, Emberly E, Fraser HB, et al. Factors underlying variable DNA methylation in a human community cohort. Proc Natl Acad Sci USA 2012;109(Suppl 2):17253-60.

39. Rampersaud GC, Kauwell GP, Hutson AD, et al. Genomic DNA methylation decreases in response to moderate folate depletion in elderly women. Am J Clin Nutr 2000;72:998-1003.

40. Unternaehrer E, Luers $\mathrm{P}$, Mill J, et al. Dynamic changes in DNA methylation of stress-associated genes (OXTR, BDNF) after acute psychosocial stress. Transl Psychiatry 2012;2:e150.

41. Baccarelli $A$, Wright RO, Bollati V, et al. Rapid DNA methylation changes after exposure to traffic particles. Am J Resp Critical Care Med 2009;179:572-8.

42. Jacobsen SC, Brøns C, Bork-Jensen J, et al. Effects of short-term high-fat overfeeding on genome-wide DNA methylation in the skeletal muscle of healthy young men. Diabetologia 2012;55:3341-9.

43. Zhong J, Colicino E, Lin X, et al. Cardiac autonomic dysfunction: particulate air pollution effects are modulated by epigenetic immunoregulation of Toll-like Recepter 2 and dietary flavonoid intake. J Am Heart Assoc 2015;4:e001423.

44. Hill $A B$. The environment and disease: association or causation? Proc Roy Soc Med 1965;58:295-300.

45. Arnson $\mathrm{Y}$, Shoenfeld $\mathrm{Y}$, Amital $\mathrm{H}$. Effects of tobacco smoke on immunity, inflammation and autoimmunity. J Autoimmun 2010;34: J258-65.

46. Hwang SJ, Ballantyne CM, Sharrett AR, et al. Circulating adhesion molecules VCAM-1, ICAM-1 and E-selectin in carotid atherosclerosis and incident coronary heart disease cases: the Atherosclerosis Risk In Communities (ARIC) study. Circulation 1997;96:4219-25.

47. Ridker PM. Role of inflammatory biomarkers in prediction of coronary heart disease. Lancet 2001;358:946-8.

48. Baccarelli A, Tarantini L, Wright RO, et al. Repetitive element DNA methylation and circulation endothelial and inflammation markers in the VA normative aging study. Epigenetics 2010;5:222-8.
49. Doran AC, Meller N, McNamara CA. Role of smooth muscle cells in the initiation and early progression of atherosclerosis. Arterioscler Thromb Vasc Biol 2008;28:812-19.

50. Blankenberg S, Barbaux S, Tiret L. Adhesion molecules and atherosclerosis. Atherosclerosis 2003;170:191-203.

51. Bielinski SJ, Pankow JS, Foster CL, et al. Circulating soluble ICAM-1 levels shows linkage to ICAM gene cluster region on chromosome 19: the NHLBI Family Heart Study follow-up examination. Atherosclerosis 2008;199:172-8.

52. Rahman A, Fazal F. Hug tightly and say goodbye: role of endothelial ICAM-1 in leukocyte transmigration. Antioxid Redox Signal 2009;11:823-39.

53. Lawson C, Wolf S. ICAM-1 signaling in endothelial cells. Pharmacol Rep 2009;61:22-32.

54. Wittchen ES. Endothelial signaling in paracellular and transcellular leukocyte transmigration. Front Biosci 2009;14:2522-45.

55. Aplin AE, Howe AK, Juliano RL. Cell adhesion molecules, signal transduction and cell growth. Curr Opin Cell Biol 1999;11: 737-44.

56. Hubbard AK, Rothlein R. Intercellular adhesion molecule-1 (ICAM-1) expression and cell signaling cascades. Free Radic Biol Med 2000;28:1379-86.

57. Wang $\mathrm{Q}$, Doerschuk CM. The signaling pathways induced by neutrophil-endothelial cell adhesion. Antioxid Redox Signal 2002;4:39-47.

58. Valinluck V, Sowers LC. Inflammation-mediated cytosine damage: a mechanistic link between inflammation and the epigenetic alterations in human cancers. Cancer Res 2007;67:5583-6.

59. Cohen S. Keynote Presentation at the Eight International Congress of Behavioral Medicine: the Pittsburgh common cold studies: psychosocial predictors of susceptibility to respiratory infectious illness. Int J Behav Med 2005;12:123-31.

60. Alt SR, Turner JD, Klok MD, et al. Differential expression of glucocorticoid receptor transcripts in major depressive disorder is not epigenetically programmed. Psychoneuroendocrinol 2010;35:544-56.

61. Weaver ICG. Epigenetic programming by maternal behavior and pharmacological intervention. Epigenetics 2007;2:22-8.

62. Dong Y, Peng CYJ. Principled missing data methods for researchers. Springerplus 2013;2:222. 